\title{
Efficient Numerical Solution of the Density Profile Equation in Hydrodynamics
}

\author{
G. Kitzhofer, ${ }^{1}$ O. Koch, ${ }^{2}$ P. Lima, ${ }^{3}$ and E. Weinmüller ${ }^{4}$
}

Received March 17, 2005; accepted (in revised form) October 4, 2005

\begin{abstract}
We discuss the numerical treatment of a nonlinear second order boundary value problem in ordinary differential equations posed on an unbounded domain which represents the density profile equation for the description of the formation of microscopical bubbles in a non-homogeneous fluid. For an efficient numerical solution the problem is transformed to a finite interval and polynomial collocation is applied to the resulting boundary value problem with essential singularity. We demonstrate that this problem is well-posed and the involved collocation methods show their classical convergence order. Moreover, we investigate what problem statement yields favorable conditioning of the associated collocation equations. Thus, collocation methods provide a sound basis for the implementation of a standard code equipped with an a posteriori error estimate and an adaptive mesh selection procedure. We present a code based on these algorithmic components that we are currently developing especially for the numerical solution of singular boundary value problems of arbitrary, mixed order, which also admits to solve problems in an implicit formulation. Finally, we compare our approach to a solution method proposed in the literature and conclude that collocation is an easy to use, reliable and highly accurate way to solve problems of the present type.
\end{abstract}

KEY WORDS: Singular boundary value problems; collocation methods; convergence; conditioning; a posteriori error estimation; adaptive mesh selection.

\footnotetext{
${ }^{1}$ Institute for Analysis and Scientific Computing, Vienna University of Technology, Wiedner Hauptstrasse 8-10, A-1040 Wien, Austria. E-mail: g.kitzhofer@kabsi.at

${ }^{2}$ Department of Mathematics, University of Tübingen, Auf der Morgenstelle 10, D-72076 Tübingen, Germany. E-mail: othmar@othmar-koch.org

${ }^{3}$ CEMAT/Department of Mathematics, Instituto Superior Tecnico, Av. Rovisco Pais, 1049001 Lisboa, Portugal. E-mail: plima@math.ist.utl.pt

${ }^{4}$ Institute for Analysis and Scientific Computing, Vienna University of Technology, Wiedner Hauptstrasse 8-10, A-1040 Wien, Austria. E-mail: e.weinmueller@tuwien.ac.at
} 


\section{INTRODUCTION}

The singular boundary value problem we discuss here originates from the Cahn-Hillard theory, which is used in hydrodynamics to study the behavior of non-homogeneous fluids. In [8], the density profile equation for the description of the formation of microscopical bubbles in a non-homogeneous fluid (in particular, vapor inside one liquid) is derived. Let us briefly recall how this equation is obtained. The state of a non-homogeneous fluid (see [8] and [10]) is described by the following system of partial differential equations:

$$
\begin{aligned}
& \rho_{t}+\operatorname{div}(\rho \vec{v})=0, \\
& \frac{d \vec{v}}{d t}+\nabla(\mu(\rho)-\gamma \Delta \rho)=0,
\end{aligned}
$$

where $\rho, \vec{v}$ denote the density and the velocity of the fluid, $\mu$ represents its chemical potential and $\gamma$ is a constant. By considering the case where the motion of the fluid is zero, the system (1), (2) is reduced to a single equation of the form

$$
\gamma \triangle \rho=\mu(\rho)-\mu_{0}
$$

where $\mu_{0}$ is a constant, depending on the state of the fluid. When searching for a solution of (3) with spherical symmetry which depends only on the variable $r$, we introduce as usual the polar system of coordinates in $\mathbb{R}^{N}$ and the equation (3) is then reduced to the following ordinary differential equation (ODE):

$$
\gamma\left(\rho^{\prime \prime}+\frac{N-1}{r} \rho^{\prime}\right)=\mu(\rho)-\mu_{0}, \quad r \in(0, \infty) .
$$

Since we consider the case of a spherical bubble, the ODE (4) is closed with the boundary conditions

$$
\rho^{\prime}(0)=0
$$

(following from spherical symmetry) and

$$
\lim _{r \rightarrow \infty} \rho(r)=\rho_{l}>0
$$

where $\rho_{l}$ is the density of the liquid surrounding the bubble. In the simplest models for non-homogeneous fluids, the chemical potential $\mu$ is a third degree polynomial, such that the difference $\mu-\mu_{0}$ has 3 real roots. 
Taking into account that $\mu\left(\rho_{l}\right)=\mu_{0}$, the right-hand side of (4) may be written in the form

$$
\mu(\rho)-\mu_{0}=4 \alpha\left(\rho-\wp_{1}\right)\left(\rho-\wp_{2}\right)\left(\rho-\rho_{l}\right), \quad 0<\wp_{1}<\wp_{2}<\rho_{l}, \quad \alpha>0 .
$$

Finally, in order to diminish the number of parameters in the equation we introduce the new variable

$$
\tilde{\rho}=\frac{\rho-\wp_{2}}{\wp_{2}-\wp_{1}}
$$

define the positive constant $\lambda=\sqrt{(\alpha / \gamma)}\left(\wp_{2}-\wp_{1}\right)$, and denote $\xi=\left(\rho_{l}-\wp_{2}\right) /$ $\left(\wp_{2}-\wp_{1}\right)>0$. Then, without loss of generality, instead of (4)-(6) we can investigate the boundary value problem

$$
\begin{aligned}
& \rho^{\prime \prime}(r)+\frac{N-1}{r} \rho^{\prime}(r)=4 \lambda^{2}(\rho(r)+1) \rho(r)(\rho(r)-\xi), \\
& \rho^{\prime}(0)=0, \quad \rho(\infty)=\xi,
\end{aligned}
$$

where, for the sake of simplicity, we write $\rho$ instead of $\tilde{\rho}$. The boundary value problem (8), (9) depends only on 3 parameters: $\lambda$, which may be chosen as $\lambda=1$ without restriction of generality, $N$ is the dimension of the problem, which in the physically meaningful case is $N=3$, and $\xi$, which is varied in the range $[0,1]$ such as to reflect different physical situations.

Note that the problem (8), (9) always has the constant solution $\rho(r) \equiv$ $\xi$, which physically corresponds to the case of a homogeneous fluid (without bubbles).

We are interested in computing a monotonously increasing solution for $0<r<\infty$, the so called "bubble-type solution". When such a solution exists it has exactly one zero $R$ in that interval, where $R$ is interpreted as the bubble radius. Furthermore, it can be shown that $-1<\rho(0)<0$ and $-1<\rho(r)<\xi, r>0$. The derivative of the solution attains a maximum at some value $\hat{r}<R$, and tends to 0 at infinity. Finally, it turns out that the solution features an interior layer, which becomes sharper for $\xi \rightarrow 1$. All these properties have been discussed in [19] (see also [18]).

It is worth to remark that the existence of a strictly increasing solution to the problem (8), (9) is far from being a simple question. In [19], it was shown (using a variational approach developed in [9]), that such a solution can exist only if $\xi$ satisfies $0<\xi<1$. Furthermore, based on the results of [11], it is possible to show that this restriction on $\xi$ is also a sufficient condition for the existence of such a solution. These results agree with the experimental evidence and the numerical simulations reported, for example, in [8]. 
In $\S 2$ of this paper, we show that a transformation of (8), (9) to a problem posed on the interval $[0,1]$ yields a well-posed boundary value problem with an essential singularity. In $\S 3$ we demonstrate that polynomial collocation can be used effectively and its classical convergence orders can be observed. Moreover, we discuss a problem formulation which yields the optimal conditioning of the associated algebraic collocation equations. Finally, we introduce a MatLab collocation code which is currently being developed for singular boundary value problems, and show that its application to (8), (9) yields a reliable and accurate solution method. In $\S 4$ we compare our approach to a solution method proposed in [19]. It turns out that collocation constitutes a more favorable solution method for problems of the present type.

\section{TRANSFORMATION TO A FINITE INTERVAL}

Here, we propose to solve (8) via transformation to a singular boundary value problem on a finite interval. To this end, two different approaches can be adopted: Transformation to a first order ODE via the Euler transformation, and subsequent transformation to the interval $[0,1]$, or transformation of the second order problem to $[0,1]$ and its direct treatment in the second order formulation. In both cases, we approximate the solution numerically using a collocation solver which is currently being developed for higher order ODEs, cf. [14].

\subsection{First Order Problem}

We apply the Euler transformation

$$
z(r):=\left(z_{1}(r), z_{2}(r)\right)^{T}=\left(\rho(r), r \rho^{\prime}(r)\right)^{T}
$$

in order to transform (8) to a first order system. Subsequently, we split the interval $(0, \infty)=(0,1] \cup[1, \infty)$, and transform the second subinterval to $(0,1]$. Consequently, we obtain the following singular boundary value problem for the unknown vector $z(s)=\left(z_{1}(s), z_{2}(s), z_{3}(s)=z_{1}(1 / s), z_{4}(s)=\right.$ $\left.z_{2}(1 / s)\right), s \in(0,1]:$

$$
z^{\prime}(s)=\left(\begin{array}{cc}
\frac{M}{s} & 0 \\
0 & \frac{-M}{s}
\end{array}\right) z(s)+\left(\begin{array}{c}
f\left(s, z_{1}(s), z_{2}(s)\right) \\
g\left(s, z_{3}(s), z_{4}(s)\right)
\end{array}\right)
$$

where

$$
M=\left(\begin{array}{cc}
0 & 1 \\
0 & 2-N
\end{array}\right)
$$




$$
\begin{aligned}
& f\left(s, z_{1}, z_{2}\right)=\left(\begin{array}{c}
0 \\
4 \lambda^{2} s\left(z_{1}+1\right) z_{1}\left(z_{1}-\xi\right)
\end{array}\right), \\
& g\left(s, z_{3}, z_{4}\right)=\left(\begin{array}{c}
0 \\
-4 \lambda^{2} \frac{1}{s^{3}}\left(z_{3}+1\right) z_{3}\left(z_{3}-\xi\right)
\end{array}\right) .
\end{aligned}
$$

The boundary conditions in the new variables read

$$
z_{2}(0)=0, \quad z_{3}(0)=\xi, \quad z_{1}(1)=z_{3}(1), \quad z_{2}(1)=z_{4}(1) .
$$

The same transformation is carried out in detail for other boundary value problems on semi-infinite intervals in [7].

In order to discuss the well-posedness of (10), (11) within the framework of singular boundary value problems developed in [12] and [13], we linearize the problem at the exact solution (using structural properties derived in [19]). This results in

$$
y^{\prime}(s)=\left(\begin{array}{cc}
\frac{N(s)}{s} & 0 \\
0 & \frac{A(s)}{s^{3}}
\end{array}\right) y(s),
$$

where

$$
\begin{aligned}
& N(s)=\left(\begin{array}{cc}
0 & 1 \\
4 \lambda^{2} s^{2}\left(3 z_{1}^{2}(s)+2(1-\xi) z_{1}(s)-\xi\right) & 2-N
\end{array}\right) \\
& A(s)=\left(\begin{array}{cc}
0 & -2 s^{2} \\
-4 \lambda^{2}\left(3 z_{3}^{2}(s)+2(1-\xi) z_{3}(s)-\xi\right) & (N-2) s^{2}
\end{array}\right) .
\end{aligned}
$$

The same boundary conditions as (11) are now posed for $y$, but with the second one replaced by the homogeneous relation $y_{3}(0)=0$. From the boundary conditions for $z$ it follows that

$$
N(0)=M, \quad A(0)=\left(\begin{array}{cc}
0 & 0 \\
-4 \lambda^{2} \xi(\xi+1) & 0
\end{array}\right) .
$$

Consequently, $y_{2}(0)=y_{3}(0)=0$ are necessary and sufficient conditions for a continuous solution of (12) to exist.

\subsection{Second Order Problem}

If instead of transforming to a first order problem, we transform (8) to the interval $[0,1]$ in the second order formulation, we obtain (cf. [16])

$$
z_{2}^{\prime \prime}(s)=\frac{N-3}{s} z_{2}^{\prime}(s)+4 \lambda^{2} \frac{1}{s^{4}}\left(z_{2}(s)+1\right) z_{2}(s)\left(z_{2}(s)-\xi\right) .
$$


Together with the original equation $(8)\left(z_{1}=\rho\right)$ and the boundary conditions

$$
z_{1}^{\prime}(0)=0, \quad z_{2}(0)=\xi, \quad z_{1}(1)=z_{2}(1), \quad z_{1}^{\prime}(1)=-z_{2}^{\prime}(1)
$$

we now need to solve a boundary value problem for a second order ODE with an essential singularity.

To check the well-posedness of this problem is not easy, since so far no theory for second-order singular problems exists. The discussion in [15] shows that this analysis is far from being trivial. However, our successful numerical computations strongly indicate that the second-order problem is also well-posed and can be treated using our techniques. In fact, this problem statement may even display certain advantages when compared to the first order formulation, see $\S 3$.

\section{NUMERICAL TREATMENT}

Here, we discuss the properties of polynomial collocation when applied to problems (10), (11), and (8), (13), (14), respectively. Collocation is a widely used and well-studied standard solution method for two-point boundary value problems, see for example [1] and the references therein. Moreover, for singular problems, many popular discretization methods like finite differences, Runge-Kutta or multistep methods show order reductions, thus making computations inefficient and prohibiting asymptotically correct error estimation and reliable mesh adaptation. A further discussion of the advantages of collocation over other numerical methods for singular boundary value problems can be found for example in [2]. For the direct solution of problems on unbounded domains, an interesting alternative is given by spectral and pseudo-spectral methods, using a basis of orthogonal functions, obtained from Chebyshev or Hermite polynomials (see [6, 20] for the first case and [21] for the latter). However, as pointed out in [20, p. 377] and in [21, p. 595], the accuracy provided by these methods depends strongly on the choice of a scaling factor, whose optimal value varies not only with the specific equation, but also with the number of basis functions. This optimal value has usually to be adjusted manually, which can be a very time-consuming process. Moreover, the choice of basis functions strongly depends on special properties of the problem at hand, as for example asymptotic behavior of the solution as the independent variable tends to infinity. Therefore we rely on collocation as a high-order, robust, general-purpose numerical method. Many of the properties of collocation methods observed for the present problem hold also in other situations where problems on a semi-infinite interval are solved 
in the formulation as an essentially singular problem on a finite interval. The general principles should therefore be useful in a broad context and the MATLAB collocation code we introduce here can be successfully applied to a variety of problems, see for example [7, 14, 16].

The numerical approximation defined by collocation is computed as follows: On a mesh

$$
\Delta:=\left\{\tau_{i}: i=0, \ldots, N\right\}, \quad 0=\tau_{0}<\tau_{1}<\cdots<\tau_{N}=1
$$

we approximate the analytical solution by a piecewise defined collocating function

$$
p(s):=p_{i}(s), \quad s \in\left[\tau_{i}, \tau_{i+1}\right], \quad i=0, \ldots, N-1,
$$

where we require $p \in C^{q-1}[0,1]$ if the order of the underlying differential equation is $q$. Here $p_{i}$ are polynomials of maximal degree $m-1+q$ which satisfy the differential equation at the collocation points

$$
\begin{aligned}
& \left\{t_{i, j}=\tau_{i}+\rho_{j}\left(\tau_{i+1}-\tau_{i}\right), i=0, \ldots, N-1, j=1, \ldots, m\right\}, \\
& 0<\rho_{1}<\cdots<\rho_{m}<1,
\end{aligned}
$$

and the associated boundary conditions are also prescribed for $p$. Classical theory, cf. [1], predicts that the convergence order is at least $O\left(h^{m}\right)$, where $h:=\max _{i}\left|\tau_{i+1}-\tau_{i}\right|$. The same could be shown in [3] and [17] for first order problems with a singularity of the first kind. For problems with an essential singularity, extensive numerical evidence and partial theoretical support indicate that the methods retain their convergence order if the collocation points are symmetric, see [5]. This behavior is also observed for the present problem, see $\$ 3.1$.

To make the computations more efficient, we additionally use an adaptive mesh selection strategy based on an a posteriori estimate for the global error of the collocation solution. We use a classical error estimate based on mesh halving. In this approach, we compute the collocation solution $p_{\Delta}(s)$ on a mesh $\Delta$. Subsequently, we choose a second mesh $\Delta_{2}$ where in every interval $\left[\tau_{i}, \tau_{i+1}\right]$ of $\Delta$ we insert two subintervals of equal length. On this new mesh, we compute the numerical solution based on the same collocation scheme to obtain the collocating function $p_{\Delta_{2}}(s)$. Using these two quantities, we define

$$
\mathcal{E}(s):=\frac{2^{m}}{1-2^{m}}\left(p_{\Delta_{2}}(s)-p_{\Delta}(s)\right)
$$

as an error estimate for the approximation $p_{\Delta}(s)$. Assume that the global error $\delta(s):=p_{\Delta}(s)-z(s)$ of the collocation solution can be expressed in 
terms of the principal error function $e(s)$,

$$
\delta(s)=e(s)\left|\tau_{i+1}-\tau_{i}\right|^{m}+O\left(\left|\tau_{i+1}-\tau_{i}\right|^{m+1}\right), \quad s \in\left[\tau_{i}, \tau_{i+1}\right],
$$

where $e(s)$ is independent of $\Delta$. Then obviously the quantity $\mathcal{E}(s)$ satisfies $\mathcal{E}(s)-\delta(s)=O\left(h^{m+1}\right)$ and the error estimate is asymptotically correct. The experimental investigations in $\$ 3.1$ below indicate that the assumption (16) is justified for the present problem.

Our mesh adaptation is based on the equidistribution of the global error of the numerical solution. Thus, we define a monitor function $\Theta(s):=$ $\sqrt[m]{\mathcal{E}(s)} / h(s)$, where $h(s):=\left|\tau_{i+1}-\tau_{i}\right|$ for $s \in\left[\tau_{i}, \tau_{i+1}\right]$. Now, the mesh selection strategy aims at the equidistribution of

$$
\int_{\tilde{\tau}_{i}}^{\tilde{\tau}_{i+1}} \Theta(s) d s
$$

on the mesh consisting of the points $\tilde{\tau}_{i}$ to be determined accordingly, where at the same time measures are taken to ensure that the variation of the stepsizes is restricted and tolerance requirements are satisfied with small computational effort. Details of the mesh selection algorithm and a proof of the fact that our strategy implies that the global error of the numerical solution is asymptotically equidistributed are given in [4].

For the solution of the boundary value problems (10), (11), and (8), (13), (14), we use a collocation solver implemented in MatLaв and equipped with the error estimate and adaptive mesh selection strategy described above, see [14]. The solver is designed for the solution of singular boundary value problems of arbitrary, mixed order, which may even be stated implicitly. A description of the implementation details and numerous examples demonstrating the favorable performance of the code are given in [14].

\subsection{Convergence and Conditioning of Collocation}

To demonstrate the favorable properties of our collocation solver when applied for the solution of the present problem, we first show that collocation when used on uniform grids retains its classical convergence order and also shows favorable conditioning of the associated algebraic equations. More extensive tests are reported in [15]. For this investigation we restrict ourselves to the choice $\xi=0.5$ in the boundary value problem (8), (9). In all our computations based on collocation, the trivial solution $\rho \equiv \xi$ is avoided by choosing the initial profile for the solution of the nonlinear collocation equations appropriately. In Table I we give 


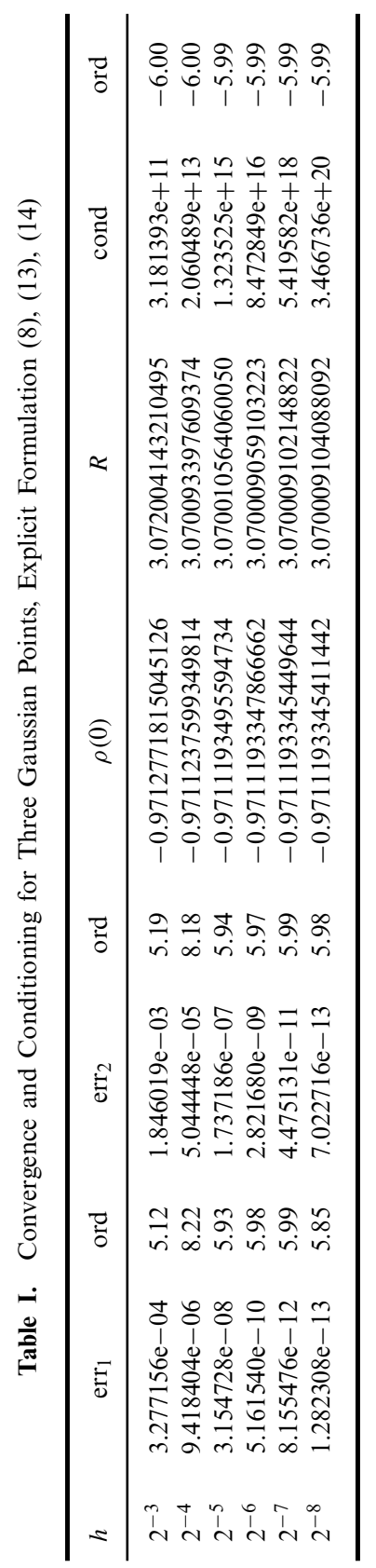


the empirical convergence orders and estimates for the condition numbers in dependence of the stepsize $h$ for the second order problem (8), (13), (14). The table displays the differences $\operatorname{err}_{j}$ for both solution components $z_{j}, j=1,2$, computed on successive meshes with uniform interval lengths $h$ and $h / 2$, respectively, and the empirical convergence orders ord computed from these. Moreover, we give estimates of the condition numbers cond of the linear systems occurring in the Newton process for the solution of the algebraic collocation equations together with their orders. Finally, the values of $\rho(0)$ and of the bubble radius $R$ are recorded for each stepsize. The computations were performed using collocation at three Gaussian points. We observe that the classical (super-)convergence order six is retained. This cannot be expected in general for problems with an essential singularity, see [5], but the present problem can obviously be solved reliably and with high accuracy. Even for the modest stepsizes in Table I the approximation quality - reflected in the values $\operatorname{err}_{j}$ and in the number of digits in $\rho(0)$ and $R$ that remain unaltered when the mesh is refined-gets close to the relative machine precision eps $\approx 1.11 \mathrm{e}-16$. However, the conditioning is unfavorable in this setting and the condition number grows as $1 / h^{6}$.

To improve the conditioning of the collocation equations, we exploit the fact that our code can deal with an implicit formulation of the differential equations. Therefore, we multiply (8) by $r$ and (13) by $s^{4}$ and repeat the computations. The results are given in Table II. In this case, the condition numbers grow as $1 / h^{2}$, which according to [1] is optimal for second order ODEs. Thus, we observe a clear dependence of the condition numbers on the order of the singular terms appearing in the equations. In the implicit formulation which is within the scope of the new code, cf. [14], the results are more favorable. However, the accuracy of the computed results does not seem strongly affected even though condition numbers differ vastly. A possible reason why the large discrepancy in condition numbers does not seem to influence the obtained accuracy is given in [22]. Still, it should be advantageous to use the implicit formulation, since bad starting values for the nonlinear equations in conjunction with bad conditioning could lead to a failure of the nonlinear solver to converge at all because the linear algebra may become unreliable in the presence of large condition numbers.

For reasons of comparison, we give the analogous results for the first order problem (10), (11), where motivated by the experience with the second order problem we again choose an implicit formulation. The convergence orders are given for the components $z_{1}, z_{3}$ corresponding to $\rho$. The accuracy in the numerical results given in Table III is very similar to that seen in Tables I and II. However, the asymptotical behavior of the condition 


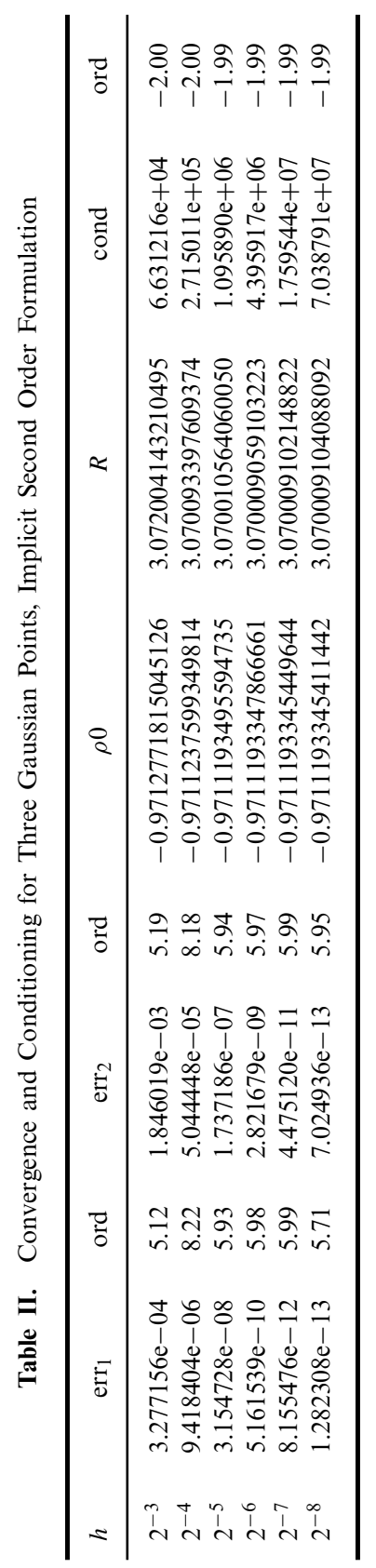




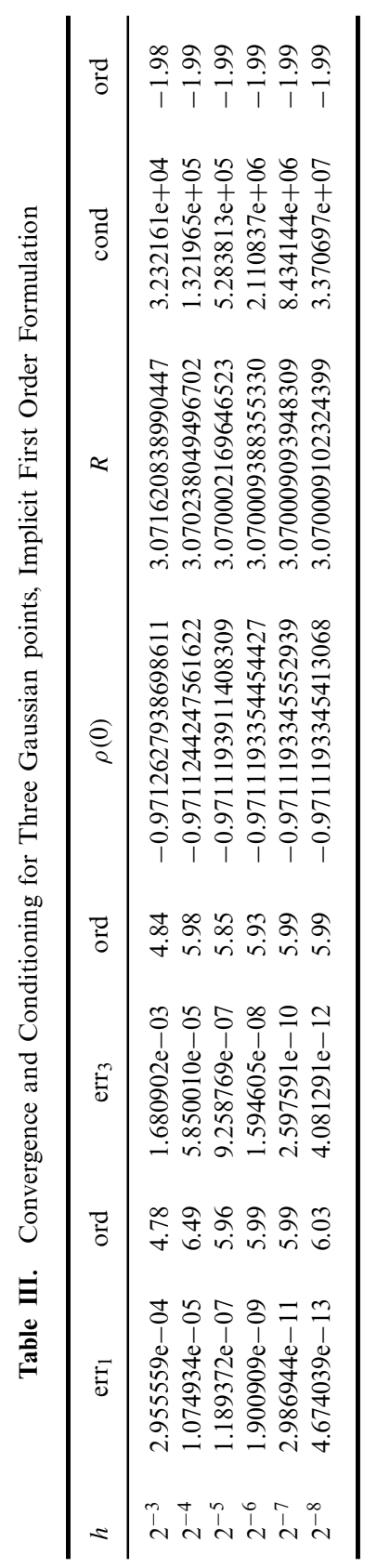


numbers is not governed by the optimal $1 / h$. Now, the condition numbers grow as $1 / h^{2}$. Again, the accuracy of the results is largely unaffected.

So we conclude that the first and second order problems admit a numerical solution with the same convergence properties and similar accuracy. However, for the second order formulation it is possible to obtain the optimal growth behavior of the condition numbers of the collocation equations by stating the problem in implicit form, which is not the case for the first order problem. On the other hand, for the formulation (10), (11), we can prove that the analytical problem is well-posed, while for second order problems with an essential singularity the respective theory is not yet developed far enough.

\subsection{Mesh Adaptation for Sharp Layers}

The solution of (8), (9) becomes computationally more challenging when the value of the parameter $\xi$ is close to 1 in contrast to the choice $\xi=0.5$ which was used in $\$ 3.1$. Even in this case, our collocation solver proved remarkably robust. By applying a simple continuation strategy to obtain starting profiles for the solution of the collocation equations as $\xi \rightarrow 1$, we could easily compute solutions for values $\xi \in(0,0.9]$. These feature an interior layer around the bubble radius $R$ which becomes sharper with growing $\xi$. The solution profiles for some values of $\xi$ are plotted in Fig. 1. For convenience of the reader, the profiles are transformed back to the original domain $[0, \infty)$ and plotted on the truncated subinterval $[0,18]$.

Particularly for the solutions with sharp layers, the computations can be sped up significantly if instead of solving the problem on a fixed grid, adaptive mesh refinement based on our a posteriori estimate of the global error is used. We illustrate this point for the case $\xi=0.8$. We solve the problem again using collocation at three Gaussian points, where a relative and absolute tolerance requirement of $1 \mathrm{e}-8$ is prescribed. Based on the favorable convergence properties of the collocation solution, the error estimate can be expected to be asymptotically correct and our mesh selection procedure should achieve asymptotical equidistribution of the global error, see also [4] and [5]. Thus, we can safely trust that the tolerances are reliably satisfied. The final mesh contains 301 points. The stepsizes are plotted as a function of the independent variable (transformed back to the original, unbounded domain) in Fig. 2. We see that indeed the smallest stepsizes occur near the interior layer at $R \approx 7$. Note that the stepsizes close to 0 appear small because of the transformation $s \rightarrow 1 / s$ used to create the plot in Fig. 2. In the actual grid resulting for (8), (13), (14), the stepsizes are quite uniform near the singularity. 


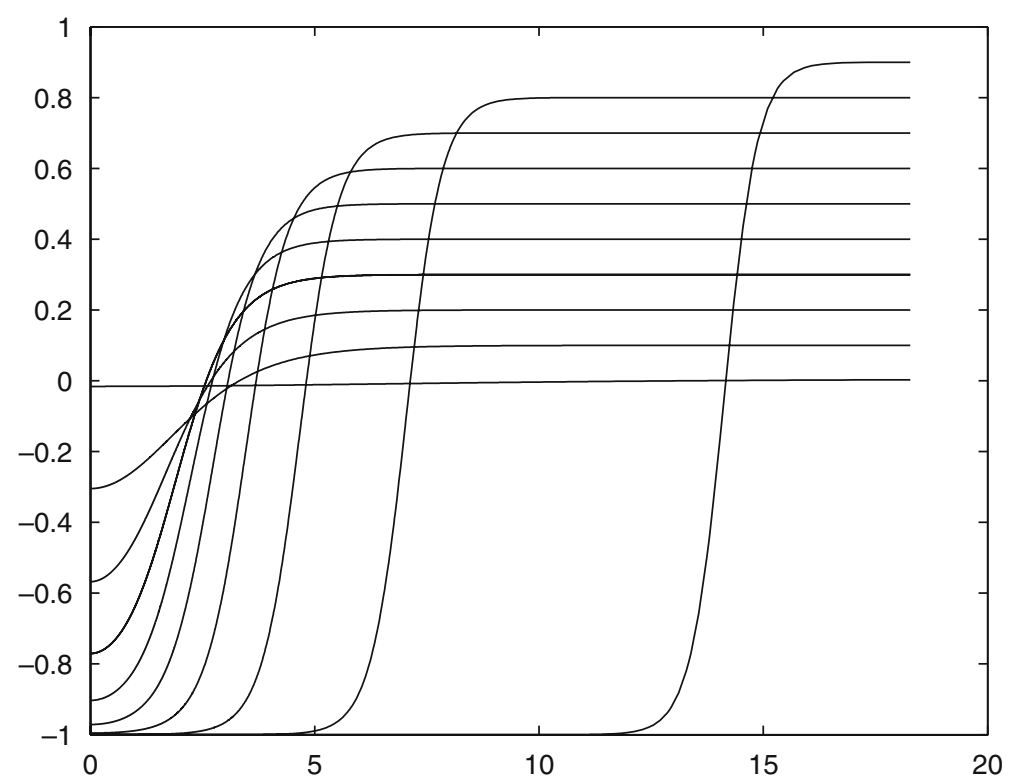

Fig. 1. Solution profiles for $\xi=0.005,0.1, \ldots, 0.9$.

To conclude this section, we give the values of $\rho(0), R$ and the integral $J$ defined by

$$
\begin{aligned}
& J=J(\rho)=\int_{0}^{\infty}\left(\frac{1}{2}\left(\rho^{\prime}(r)\right)^{2}+W(\rho(r))\right) r^{N-1} d r, \\
& W(\rho)=4 \lambda^{2} \int_{\xi}^{\rho}(s+1) s(s-\xi) d s,
\end{aligned}
$$

for different values of $\xi$. The integral $J(\rho)$ defines a functional, for which the boundary value problem (8), (9) represents the Euler-Lagrange equation, in the following sense: this integral converges if and only if $\rho$ is a solution of (8), (9) (see [19], Eq. (3.2)).

Again, collocation at three Gaussian points was used and absolute and relative tolerances $1 \mathrm{e}-8$ have been satisfied. Only in the case $\xi=0.9$ we used five Gaussian points to achieve the desired accuracy with acceptable computational effort. To compute the value of $J$, we transform $J$ to the interval $[0,1]$ and use numerical quadrature of the transformed integral evaluated at our numerical solution. To this end we truncate the integration interval to $[0.002,1]$ to avoid the evaluation at 0 and use the adaptive quadrature routine quadl which is part of MATLAB 7.0 (R14). The tolerance for the quadrature is set to $1 \mathrm{e}-10$, so that we can expect an 


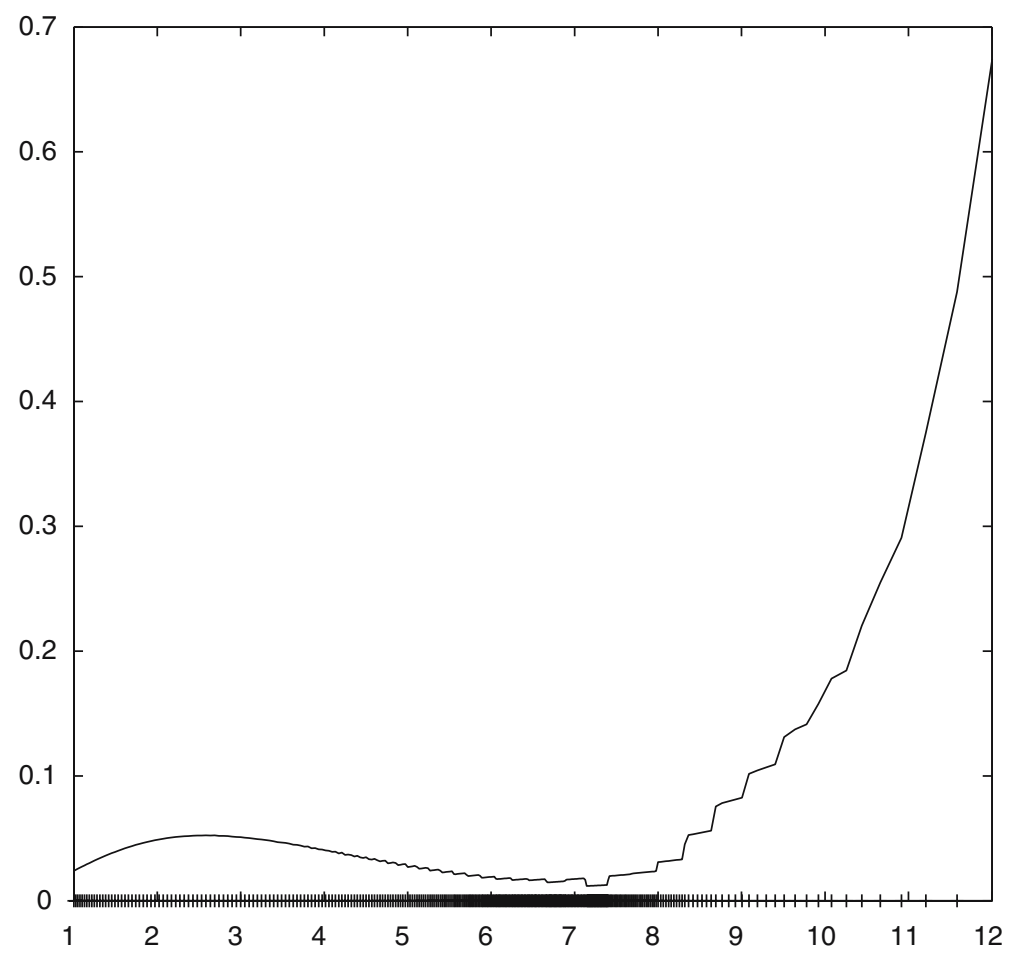

Fig. 2. Stepsizes generated by mesh adaptation for $\xi=0.8$.

overall accuracy of about 8 digits for the values of $\rho(0), R$ and $J$. This is also supported by comparing the results with the computations on uniform meshes, even the first ten digits are the same as those for the most precise results given in Table II.

\section{COMPARISONS}

In this section, we describe an alternative approach for the numerical solution of (8), (9) proposed in [19]. A comparison of the results with the collocation methods described in $\$ 3$ indicates that collocation can be applied to the problem more straightforwardly, and with better control of the accuracy of the solution.

The approach developed in [19] is based on a stable shooting method, which uses the asymptotic properties of the solution near the singularities to solve the boundary value problem. More precisely, it is shown in [19] that equation (8) possesses a one-parameter set of solutions which satisfy 
the condition $\rho^{\prime}(0)=0$ and that each solution in this set may be expanded in a series of the form

$$
\rho(r)=\rho_{0}+\sum_{k=1}^{\infty} \rho_{2 k}\left(\rho_{0}\right) r^{2 k}, \quad 0<r \leqslant \delta,
$$

where $\rho_{0}$ is the parameter of the set, $\rho_{2 k}$ are coefficients which are independent of $r$ and can be computed recursively. Moreover, this method uses the fact that there is another one-parameter set of solutions of equation (8) which satisfy the boundary condition $\rho(\infty)=\xi$ and each solution in this set may be expanded in a series of the form

$$
\rho(r)=\xi+\frac{1}{r^{(N-1) / 2}} \sum_{k=1}^{\infty} C_{k}(r) b^{k} e^{-k r \tau}, \quad r \geqslant r_{\infty},
$$

where $\tau=2 \lambda \sqrt{\xi(\xi+1)}$ and $b<0$ is the parameter of the set. $C_{k}$ are functions of $r$ which may be determined by substituting (20) into equation (8) and solving the resulting Cauchy problems. In particular, in the case $N=3$, it may be shown that $C_{1}(r) \equiv 1$ and this was the only term of the series (20) which was used to obtain the numerical results in [19].

Since the considered boundary value problem has singularities at both endpoints and the singularity at infinity is a saddle point, it is not possible to solve the problem numerically just by shooting from one of the endpoints. Therefore, according to the method presented in [19] the interval $\left[\delta, r_{\infty}\right]$ (where the numerical solution of the problem is sought) is divided into two subintervals $\left[\delta, r_{0}\right]$ and $\left[r_{0}, r_{\infty}\right]$ and one auxiliary boundary value problem is solved on each sub-interval. By adding the boundary condition $\rho\left(r_{0}\right)=0$ to each of these auxiliary problems, they can be solved by standard shooting methods, making use of the series (19) and (20) to approximate the solution at $\delta$ and $r_{\infty}$, respectively. Due to the condition imposed on the solution of the auxiliary problems, the global solution has a root at $r_{0}$, which excludes the trivial solution $\rho \equiv \xi$. Moreover the solution of each auxiliary problem must satisfy matching conditions at $r_{0}$ which assure that the derivative of the global solution is continuous on the whole interval $\left[\delta, r_{\infty}\right]$. This algorithm was encoded in Mathematica and the command NDSolve of this software was used to solve all the involved initial value problems.

The results obtained by this computational method are displayed in Table V. These results were obtained using the absolute tolerance $\epsilon=10^{-14}$ when applying the shooting method to each auxiliary boundary value problem. The values of $\delta$ and $r_{\infty}$ were chosen in the range $\left[10^{-5}, 10^{-3}\right]$ and $[7,11]$, respectively, depending on $\xi$. The value of $\delta$ seems not to 
affect the accuracy of the method substantially, at least if it is within the mentioned range. Concerning the value of $r_{\infty}$, for each value of $\xi$, there is an optimal value of this parameter which provides the maximal accuracy. This optimal value (given in the last column of the table) had to be found experimentally: $r_{\infty}$ was increased until the best possible correspondence with the results in Table IV was achieved.

We observe that the values for $\rho(0)$ and $R$ in Table $\mathrm{V}$ correspond to the results given in Table IV up to about seven or eight digits in general (we will discuss the less accurate results for $\xi=0.1,0.2$ below). Since these last results are expected to be at least eight digits accurate, we conclude that the precision achieved by collocation seems slightly more satisfactory at this moment.

Thus, for $\rho(0)$ and $R$ it is possible to achieve acceptable accuracy, while the results for $J$ clearly favor the methods from $\S 3$. It should be stressed that there is no way to control the error reliably using the algorithm described in this section, in contrast to the collocation code. Rather,

Table IV. Values of $\rho(0)$, the Bubble Radius $R$ and the Integral $J$, Computed to Accuracy $1 \mathrm{e}-8$ for $\xi=0.1, \ldots, 0.9$

\begin{tabular}{lccl}
\hline$\xi$ & $\rho(0)$ & \multicolumn{1}{c}{$R$} & \multicolumn{1}{c}{$J$} \\
\hline 0.1 & -0.3046629135732414 & 3.321895965371974 & 0.06423770835737890 \\
0.2 & -0.5677637625604254 & 2.685731317498248 & 0.2173942457657418 \\
0.3 & -0.7707036640095047 & 2.582331278595824 & 0.4892016493829168 \\
0.4 & -0.9031250928082184 & 2.720986514572336 & 0.9716918685380278 \\
0.5 & -0.9711193345575906 & 3.070009104270134 & 1.884169391417022 \\
0.6 & -0.9953000352054564 & 3.695885728381596 & 3.774049025090401 \\
0.7 & -0.9997788979967360 & 4.816886914011474 & 8.316634452859129 \\
0.8 & -0.9999995735610752 & 7.130991634527193 & 22.63550082997492 \\
0.9 & -0.9999999999999973 & 14.16875522250826 & 107.4896967510244 \\
\hline
\end{tabular}

Table V. Values of $\rho(0)$, the Bubble Radius $R$ and the Integral $J$ Defined by (17), $\xi=0.1, \ldots, 0.8$

\begin{tabular}{|c|c|c|c|c|}
\hline$\xi$ & $\rho(0)$ & $R$ & $J$ & $r_{\infty}$ \\
\hline 0.1 & -0.304745 & 3.32172 & 0.06349 & 7 \\
\hline 0.2 & -0.567765 & 2.68575 & 0.21340 & 7 \\
\hline 0.3 & -0.7707036967 & 2.582233132 & 0.48450 & 8 \\
\hline 0.4 & -0.9031250938 & 2.720986519 & 0.9691 & 8 \\
\hline 0.5 & -0.9711193346 & 3.070009102 & 1.884 & 9 \\
\hline 0.6 & -0.9953000354 & 3.695888572 & 3.696 & 9 \\
\hline 0.7 & -0.9997788981 & 4.816886906 & 8.315 & 10 \\
\hline 0.8 & -0.9999995737 & 7.130991636 & 22.611 & 11 \\
\hline
\end{tabular}


parameters were manually adjusted until good correspondence with the reliable results from Table IV was observed.

In the presence of the sharp layer for $\xi=0.9$, the algorithm described in this section failed to produce any useful results at all. This algorithm is not applicable either for values of $\xi$ less than 0.3. The values given in Table $\mathrm{V}$ for $\xi=0.1$ and $\xi=0.2$ were obtained using a modification of this algorithm, described in [19]. The accuracy of these results appears to be only about four digits in general.

Finally, the computational effort necessary when using the algorithm from [19] is considerable. Even though it is not possible to assess details of the performance because blackbox solvers from MathEMATICA were used to integrate the initial value problems occurring in the course of the shooting procedure described above, we can provide an impression of the required computational effort by noting that to compute the results for $\xi=0.5$ with $\delta=10^{-5}, r_{\infty}=9$ it was necessary to solve 573 Cauchy problems on $[\delta, 1]$ and 481 Cauchy problems on $\left[1, r_{\infty}\right]$. This complexity definitely exceeds the one necessary for the collocation approach discussed in $\S 3$.

\section{CONCLUSIONS}

In this paper, we have presented a MATLAB collocation code which is currently being developed for implicit, mixed order singular boundary value problems in ordinary differential equations. As a first example to show the advantageous properties of the solver, the density profile equation from hydrodynamics was solved. After demonstrating how the problem can be expressed as a well-posed singular boundary value problem on a finite interval, we showed that the implemented collocation methods retain their classical convergence orders. Also, optimal conditioning of the collocation equations can be achieved by posing the differential equations in an implicit form. We also showed that using an a posteriori error estimate and adaptive mesh selection yields reliable error control and meshes that are denser where the solution varies strongly. Finally, comparisons with a solution method previously presented in the literature support the proposition that our collocation code is comparably straightforward to use and represents an improvement in reliability and accuracy. These favorable properties can be expected to prevail for a large class of singular boundary value problems, see for instance $[7,14,16]$.

\section{ACKNOWLEDGMENTS}

The work described in this paper was supported in part by SFB 382 at the University of Tübingen (Germany), by project P 17253-N12 
of the Austrian Science Fund FWF at Vienna University of Technology (Austria), and by project POCTI/MAT/45700/2002 at Instituto Superior Técnico in Lisbon (Portugal).

\section{REFERENCES}

1. Ascher, U., Mattheij, R. M. M., and Russell, R. D. (1988). Numerical Solution of Boundary Value Problems for Ordinary Differential Equations, Prentice-Hall, Englewood Cliffs, NJ.

2. Auzinger, W., Koch, O., and Weinmüller, E. (2002). Efficient collocation schemes for singular boundary value problems. Numer. Algorithms 31, 5-25.

3. Auzinger, W., Koch, O., and Weinmüller, E. (2005). Analysis of a new error estimate for collocation methods applied to singular boundary value problems. SIAM J. Numer. Anal. 42, 2366-2386.

4. Auzinger, W., Koch, O., and Weinmüller, E. (2005). Efficient mesh selection for collocation methods applied to singular BVPs. J. Comput. Appl. Math. 180, 213-227.

5. Auzinger, W., Koch, O., and Weinmüller, E. (2004). Collocation methods for boundary value problems with an essential singularity. In Lirkov, I., Margenov, S., Wasniewski, J., and Yalamov, P. (eds.), Large-Scale Scientific Computing, Lecture Notes in Computer Science, Vol. 2907, Springer Verlag, pp. 347-354.

6. Boyd, J. P. (1987). Spectral methods using rational basis functions on an infinite interval. J. Comput. Phys. 69, 112-142.

7. Budd, C. J., Koch, O., and Weinmüller, E. (2004). Self-similar blow-up in nonlinear PDEs, AURORA TR-2004-15, Inst. for Anal. and Sci. Comput., Vienna University of Technology, Austria. Available at http://www.vcpc.univie.ac.at/aurora/publications/.

8. Dell'Isola, F., Gouin, H., and Rotoli, G. (1996). Nucleation of spherical shell-like interfaces by second gradient theory: numerical simulations. Eur. J. Mech. BlFluids 15, 545568.

9. Derrick, G. (1965). Comments on nonlinear wave equations as models for elementary particles. J. Math. Phys. 5, 1252-1254.

10. Gavrilyuk S. L., and Shugrin, S. M. (1996). Media with equations of state that depend on derivatives. J. Appl. Mech. Tech. Phys. 37, 177-189.

11. Gazzola, F., Serrin, J., and Tang, M. (2000). Existence of ground states and free boundary problems for quasilinear elliptic operators. Adv. Differential Equations. 5, 1-30.

12. de Hoog, F. R., and Weiss, R. (1976). Difference methods for boundary value problems with a singularity of the first kind. SIAM J. Numer. Anal. 13, 775-813.

13. de Hoog, F. R., and Weiss, R. (1980). On the boundary value problem for systems of ordinary differential equations with a singularity of the second kind. SIAM J. Math. Anal. 11, 41-60.

14. Kitzhofer, G. (2005). Numerical treatment of implicit singular BVPs, Ph.D. Thesis, Inst. for Anal. and Sci. Comput., Vienna University of Technology, Austria. In preparation.

15. Kitzhofer, G., Koch, O., and Weinmüller, E. (2004). Collocation methods for the computation of bubble-type solutions of a singular boundary value problem in hydrodynamics, Techn. Rep. ANUM Preprint Nr. 14/04, Inst. for Anal. and Sci. Comput., Vienna Univ. of Technology, Austria. Available at http://www.math.tuwien.ac.at/ inst115/preprints.htm.

16. Kitzhofer, G., Koch, O., and Weinmüller, E. (2004). Kollokationsverfahren für singuläre Randwertprobleme zweiter Ordnung in impliziter Form, Techn. Rep. ANUM Preprint Nr. 9/04, Inst. for Anal. and Sci. Comput., Vienna Univ. of Technology, Austria. Available at http://www.math.tuwien.ac.at/ inst115/preprints.htm. 
17. Koch, O. (2005). Asymptotically correct error estimation for collocation methods applied to singular boundary value problems. Numer. Math. 101, 143-164.

18. Lima, P. M., Chemetov, N. V., Konyukhova, N. B., and Sukov, A. I. Analytical-numerical approach to a singular boundary value problem, Proceedings of CILAMCE XXIV, Ouro Preto, Brazil.

19. Lima, P. M., Konyukhova, N. B., Sukov, A. I., and Chemetov, N. V. (2006). Analyticalnumerical investigation of bubble-type solutions of nonlinear singular problems. J. Comput. Appl. Math. 189, 260-273.

20. Liu, Y., Liu, L., and Tang, T. (1994). The numerical computation of connecting orbits in dynamical systems: a rational spectral approach. J. Comput. Phys. 111, 373-380.

21. Tang, T. (1993). The Hermite spectral method for Gaussian-type functions. SIAM J. Sci. Comput. 14, 594-606.

22. Tang T., and Trummer, M. (1996). Boundary layer resolving pseudospectral methods for singular perturbation problems. SIAM J. Sci. Comput. 17, 430-438. 